\title{
The response of stratified swirling flames to acoustic forcing: experiments and comparison to model
}

\author{
Zhiyi Han*, Simone Hochgreb \\ University of Cambridge Department of Engineering, Trumpington Street Cambridge, \\ United Kingdom, CB2 1PZ
}

\begin{abstract}
The gradient of local equivalence ratio in reacting mixtures significantly affects the flame structure and their corresponding response to acoustic velocity perturbations. We study the effect of acoustic velocity fluctuations on flames created by two co-annular, swirling streams with different equivalence ratios to simulate the effects of pilot-mains split. The flames are stabilized both by a bluff body and by swirl. The flame responses were measured via chemiluminescence as a function of frequency, in the linear perturbation range. A linearized version of the $G$-equation model is employed to describe the flame dynamics, combined with effects of axial and azimuthal velocity perturbations downstream of the swirlers. The model accounts for the phase shift between the main acoustic and swirler vortical perturbations, which propagate at different speeds. The very different flame structures generated by different fuel splits lead to different flame responses. Models based on time delay of vortical disturbances are able to capture the behaviour reasonably well for the case of outer fuel enrichment, but offer limited agreement for the case of the inner enriched flame, particularly under higher mean equivalence ratios.
\end{abstract}

Keywords: Combustion instabilities, Stratified turbulent flame, Flame transfer function, $G$-equation

\footnotetext{
${ }^{*}$ Corresponding author

Email address: zh253@cam.ac.uk (Zhiyi Han)
} 


\section{Introduction}

Combustion instabilities arise in confined systems from a positive coupling between pressure perturbations and the heat release rate oscillation [1], and have posed significant challenges to the development of advanced gas turbine engines [1-3]. The drive towards lean operation leaves combustion systems more susceptible to acoustic instability and blow off, as the heat release zone is more compact, and more sensitive to air and/or fuel perturbations [4]. A compromise is often made by operating a richer pilot zone surrounded by a leaner mixture. We investigate how such systems respond to acoustic perturbations.

The main drivers of combustion instabilities are fluctuations in acoustic velocity $[5,6]$ and the corresponding changes in equivalence ratio [7-9]. Most previous investigations have been focused on premixed flames, both experimentally and computationally, including laminar $[3,5]$ and turbulent flames [10]. In general the response of the flame is higher at lower frequencies than that at higher frequencies, and the rate of heat release fluctuation (usually as evidenced from chemiluminescence) is linear up to a threshold amplitude, beyond which non-linearities arise. The latter are often associated with a significant change in flame shape, by altering the relative phasing of heat release rate and pressure. These non-linearities are usually responsible for determining the end state of the perturbation cycle. The principal difficulty in making predictions of the onset of instabilities lies in establishing an effective model for the flame response as a function of frequency and perturbation amplitude.

Equivalence ratio fluctuations often occur due to imperfect mixing in fuel and air. The combined effects of velocity and equivalence ratio have been investigated in [11]. Scarinci et al.[12] have shown it is possible to decouple the fuel-air mixing process from pressure pulsations in the combustion chamber such that a phased mixing allows the spreading of the time delays of air and fuel fluctuations. Kim and Hochgreb showed experimentally how radial gradients in equivalence ratio simulating pilot-mains distribution can affect flame response $[13,14]$. These investigations clearly demonstrate the role of phase lag between pressure and equivalence ratio perturbations in determining instabilities.

Acoustic models of combustion systems use network elements characterized by transfer functions [15]. The response of flames to acoustic perturbations in these models is often represented by a flame transfer function (FTF), 
detailing the gain and phase difference of normalized heat release rate oscillation as function of velocity perturbation. These functions have also been extended into the non-linear range via a flame describing function (FDF) [16-19]. These models use a combination of physically base models and adjustable parameters to describe and understand the physical mechanisms that control the flame dynamic response. Many of the theoretical models have relied on the kinematic $G$-equation, in which the flame surface is presented as an isosurface separating the fresh from burned gases, which moves subject to the action of acoustic waves. Schuller et al. [20] have created a unified framework dealing with conical and V-shaped laminar flames based on this method. Palies et al. [21] extended the model to premixed swirling flames, by suitably accounting for the convected perturbation induced by the swirler. The latter showed that the response of swirling flames is a result of the combined effects of axial and azimuthal velocity components.

In this paper we investigate the influence of an imposed radial split in fuel distribution across the injector on the flame transfer function turbulent flames, at various global inlet conditions. A $G$-equation model adapted to the current conditions is used to understand and explain the response of the flames. We describe the experiment, the model and discuss the comparison and limitations of the latter.

\section{Experimental methods}

The measurements were performed using an axisymmetric burner shown in Fig. 1. An upstream mixing section consists of two concentric tubes (diameters: 15.80 and $27.75 \mathrm{~mm}$, thickness $1.5 \mathrm{~mm}$ ) and a centerbody $(6.35$

$\mathrm{mm}$ ), which acts as a flame stabilizer. Metered air and fuel mixture is split to flow in two directions: directly to the plenum, or via a graduated ball valve to the siren, to allow control of the amplitude of the acoustic oscillation. The siren consists a stator and a rotating plate, which is controlled by a variablespeed motor (EZ motor Model 55EZB500). Additional fuel is injected about $1000 \mathrm{~mm}$ upstream of the combustor chamber entrance into either inner or outer streams to create the desired radial equivalence ratio difference in stratified flames. The volumetric flow rates of both air and fuel are controlled by Alicat mass flow controllers (Air: 0-2000 slpm; Methane: 0-50 slpm \& 020 slpm; MCR/MC Series, $\pm 0.2 \%$ FS accuracy). Two axial swirlers (six vanes of $0.50 \mathrm{~mm}$ thickness and $20.0 \mathrm{~mm}$ length, angled at 45 degrees to the flow direction, swirl number 0.55 ) in both the inner and outer tube provide 
additional flame stability. The outer and inner swirlers end 30.0 and 10.0 $\mathrm{mm}$ from the outlet, respectively. The tube ends at a steel plate flush to the combustor inlet. The combustor is an optically accessible fused-silica tube (diameter: $95.0 \mathrm{~mm}$, length: $150.0 \mathrm{~mm}$ ). The modes are decoupled by appropriate design of the lengths and cross sectional areas of the tubes. All experiments were conducted at ambient temperature and pressure conditions $\left(T=20 \pm 2{ }^{\circ} \mathrm{C}, p=1 \pm 0.02\right.$ bar $)$.

The bulk velocity entering the combustor is $5 \mathrm{~m} / \mathrm{s}$. The corresponding Reynolds numbers are approximately 3000 based on this velocity and the hydraulic diameter of each annulus. The stratification ratio $(S R)$ is represented by the ratio of equivalence ratios of the inner to the outer stream, whilst keeping the total power constant. The premixed case, $S R=1.0$, was chosen as baseline for comparison with two stratified cases: $S R=0.5$ (outer enrichment) and $S R=2.0$ (inner enrichment). Three global equivalence ratios of 0.6, 0.7 and 0.8 were tested, with total powers of 4.9-6.5 kW. The conditions for the investigated flames are listed in Table 1. Pressures and acoustic velocities are measured by two pressure transducers (40GP GRAS) spaced $20 \mathrm{~mm}$ upstream of the swirlers. Acoustic velocities are also measured by a hot film probe (Standard Model 55P11, Dantec) controlled by a constant temperature anemometer (CTA) bridge (Mini CTA 54T40, Dantec) mounted $20 \mathrm{~mm}$ upstream of the inlet. All data were logged via a data acquisition system (National Instruments, BNC-2111) at a sampling frequency of $8192 \mathrm{~Hz}$. A total of 32768 data points were taken for each test point, resulting in a frequency resolution of $0.25 \mathrm{~Hz}$ and a time resolution of $0.122 \mathrm{~ms}$. A photomultiplier (Thorlabs PMM01) was used to capture the global $\mathrm{OH}^{*}$ $(307 \pm 5 \mathrm{~nm})$ chemiluminescence signals as direct indicator of heat release rate of the flames via bandpass filters (FGUV11 (275-375 nm), Thorlabs). Previous investigations have shown that $\mathrm{OH}^{*}$ chemiluminescence emission intensities depend linearly on the air/fuel mixture mass flow rate and as a power law on the equivalence ratio $\Phi$ of the flame [22]. However, in partially premixed flames, chemiluminescence can be a nonlinear function of the total heat release rate. Those nonlinearities are taken into account when mapping fluctuations in chemiluminescence to those of heat release rate. It is assumed that during forcing, the captured chemiluminescence still follows the same relation to heat release as when unforced.

A high speed CMOS camera (Photron FASTCAM-SA1.1) captures the $\mathrm{OH}^{*}$ chemiluminescence images. A gated intensifier (UVi2550-10S20, Invisible Vision) and an objective lens (Nikon Rayfact UV-105 mm f/4.5) are 
installed in front of the camera, and a bandpass filter (FGUV11, Thorlabs) records direct light emission. The image dimensions are $94 \mathrm{~mm}$ x $128 \mathrm{~mm}$, corresponding to $752 \times 1024$ pixels. A total of 4096 images were acquired at an exposure time of $50 \mu \mathrm{s}$ and an acquisition rate of 8000 frames/second. A background image is subtracted from the raw images, followed by total averaging to generate a line-of-sight on flame structure. Three-point Abeldeconvolution [23] is used to extract two-dimensional information from the time-averaged images. The deconvoluted images shown are weighted by the local radius to remove the noise around the centerline; this leads to a linear increase in the signal with radius in the images displayed. All images shown are self-scaled to the maximum in the set at the particular condition.

\section{Flame response model}

\subsection{Swirl flame perturbation model}

The response of flames to acoustic perturbations is usually characterized by a flame transfer function $F$ which relates the gain and phase of the heat release rate perturbation as function of the frequency and intensity of the incoming velocity perturbation:

$$
F(\omega)=\lim _{A \rightarrow 0} \frac{\hat{Q} / \bar{Q}}{\hat{u} / \bar{u}}=\lim _{A \rightarrow 0} \mathcal{G} e^{-i \omega \tau}
$$

where the complex gain $\mathcal{G}$ and phase difference $\theta=\omega \tau$ are also functions of the forcing angular frequency $\omega$, as well as amplitude, $A=\hat{u} / \bar{u}$. The variable $Q$ is the heat release rate per unit cross sectional area, and $u$ represents the incoming velocity. The notations $\left(^{\wedge}\right)$ and $\left(^{-}\right)$denote the Fourier transform of a time signal and the average signal, respectively. The flame is assumed to be instantaneously described as a surface which separates the reactants and products by the kinematic $G$-equation [21, 24]:

$$
\frac{\partial G}{\partial t}+\mathbf{u} \cdot \nabla G=S_{T}|\nabla G|
$$

where $\mathbf{u}$ is the local convective flow velocity and $S_{T}$ is the turbulent burning velocity. Equation (2) can be linearised about average values denoted by subscript 0 , with fluctuations denoted by subscript 1, to yield [21]:

$$
\begin{aligned}
& \frac{\partial G_{1}}{\partial t}+\left(\mathbf{u}_{\mathbf{0}}+S_{T_{0}} \mathbf{n}\right) \cdot \nabla G_{1} \\
= & {\left[\mathbf{u}_{\mathbf{1}} \cdot \mathbf{n}-\frac{S_{T_{1}}}{S_{T_{0}}} \mathbf{u}_{\mathbf{0}} \cdot \mathbf{n}\right]\left|\nabla G_{0}\right| }
\end{aligned}
$$


where the normal vector $\mathbf{n}=-\nabla G_{0} /\left|\nabla G_{0}\right|$. The flame motion becomes controlled by the perturbation of flow velocity and turbulent flame velocity. The total flame transfer function for the turbulent flame, $F^{T}(\omega)$ can be expressed as combination of perturbation of both axial acoustic velocity and turbulent burning velocity, assuming that:

$$
\frac{\hat{Q}_{T}}{\bar{Q}_{T}}=\frac{\hat{Q}_{u}}{\bar{Q}_{u}}+\frac{\hat{Q}_{S_{T}}}{\bar{Q}_{S_{T}}}
$$

Assuming that the perturbation velocity is proportional to the perturbation in turbulent burning velocity, we have $\mathbf{u}_{\mathbf{1}}=S_{T_{1}} / S_{T_{0}} \mathbf{u}_{\mathbf{0}}$ to yield:

$$
\frac{\hat{Q}_{S_{T}}}{\bar{Q}_{S_{T}}}=-F^{L}(\omega) \frac{\hat{S}_{T_{1}}}{S_{T_{0}}}
$$

which leads to:

$$
F^{T}(\omega)=F^{L}(\omega)\left[1-\frac{\hat{S}_{T_{1}} / S_{T_{0}}}{\hat{u} / \bar{u}}\right]
$$

where $F^{L}(\omega)$ is the transfer function defined for the corresponding laminar flame [20], but adapted here to the turbulent flame with burning velocity $S_{T_{0}}$. The expression of $F^{L}(\omega)$ is given in $[20,25]$ for a conical $\mathrm{V}$-shaped flame:

$$
\begin{array}{r}
F^{L}(\omega)=\frac{2}{\omega_{*}^{2}}\left(\frac{1}{1-\cos ^{2} \alpha}\right)\left[\exp \left(i \omega_{*}\right)-1\right. \\
\left.-\frac{\exp \left(i \omega_{*} \cos ^{2} \alpha\right)-1}{\cos ^{2} \alpha}\right] \\
+\frac{2}{i \omega_{*}}\left(\frac{1}{1-\cos ^{2} \alpha}\right)\left[\exp \left(i \omega_{*} \cos ^{2} \alpha\right)-\exp \left(i \omega_{*}\right)\right]
\end{array}
$$

where $\omega_{*}=\omega R / S_{T_{0}} \cos \alpha$ is the normalized angular velocity, $R$ the radius of the centroid of the flame, and $\alpha$ is the flame angle. In the present case with two streams at different equivalence ratios, different values for the flame speed and angle must be used for each branch of the flame.

The relationship between the turbulent burning velocity fluctuation and the incoming velocity modulations is still unknown. However, a recent study by Shin and Lieuwen [26] showed that the forced turbulent burning velocity 
fluctuations can be related to ensemble averaged curvature of the flame. In the present work, a simplified linearized model is used, from [21]:

$$
\frac{\hat{S}_{T_{1}}}{S_{T_{0}}}=\chi \frac{\hat{u}_{\varphi}}{\bar{u}_{\varphi}}+\zeta \frac{\hat{u}_{x}}{\bar{u}_{x}}
$$

where $u_{\varphi}$ is the azimuthal component and $u_{x}$ is the axial component of the flow. The relationship between velocity fluctuations in the azimuthal and axial directions have been discussed in [25], and are based on the analysis of incident acoustic waves interacting with a row of blades by Cumpsty and Marble [27]. The axial velocity perturbation propagates at the speed of sound, while the azimuthal velocity fluctuation travels at the mean convective speed. As the acoustic perturbation impinges onto the swirlers, a time delay arises between the acoustic time and a time associated with the transport of the vortical disturbance arising at the swirler vanes, which is represented as an associated phase shift:

$$
\frac{\hat{u}_{\varphi}}{\bar{u}_{\varphi}}=\frac{\hat{u}_{x}}{\bar{u}_{x}} \exp \left(i \theta_{s}\right)
$$

Since these perturbations only depend on flow, the coefficients $\chi, \zeta$ and $\theta_{s}$ are fixed for all cases, and best fit according to the original model [25]; in the present work, $\theta_{s}$ is calculated from the relative transit time across the swirler based on geometry. The distance to be traveled by either perturbation is comprised of the total distance from the inlet of the entrance of the combustor to the centroid of heat release of flame (based on chemiluminescence), $L_{f}$, plus the distance from the combustor to the swirler, $l_{s}$. The acoustic perturbation travels at the speed of sound $c$, whereas the vortical perturbation arising at the swirler travels at the mean convective velocity $\bar{u}$. The difference in travel time $\tau$ and the corresponding phase $\theta_{s}$ are then:

$$
\begin{array}{r}
\tau=\left(L_{f}+l_{s}\right)\left(\frac{1}{U}-\frac{1}{c}\right) \\
\theta_{s}=\tau \omega+\theta_{s 0}
\end{array}
$$

where $\theta_{s 0}$ is the extrapolated phase shift at zero frequency. The resulting expression for the transfer function is therefore:

$$
F^{s}(\omega)=F^{L}(\omega)\left[1-\left(\zeta+\chi \exp \left(i \theta_{s}\right)\right)\right]
$$

The parameters $\chi, \zeta$ and $\theta_{s 0}$ are constants for all experiments, whereas $L_{f}$ and $\alpha$ are obtained from the images for each flame. 


\subsection{Geometric flame parameters}

The original model for the flame perturbation was applied to a conical V-flame. The current flame can have more than one stabilization point, depending on operating conditions. We have therefore adapted the present model to accommodate the various multiple stabilization points derived from the flame images, and illustrated schematically in Fig. 2. For an A-shaped flame, we have two legs anchoring the flame at the edge of the bluff body and either the rim of the dump plane or the splitter tube. An M-flame has three attachment points, with an additional attachment to the splitter tube edge. Finally, a D-flame corresponds to a V-shaped flame anchored at the bluff body, but with a large vortical region extending to the corner recirculation zone. These are of course crude geometrical approximations corresponding to the mean flame shapes at the different conditions, but they allow a sensible analysis of the features and sensitivities to perturbation of the flame area. For the A-shaped flame, the flame length $L_{f}$ and flame angle $\alpha$ are determined according to the location of centroid of heat release rate, obtained from the chemiluminescence signal. In the M-shaped flame, there are two mixtures at different equivalence ratios. We assume that the ratio of the base turbulent flame speeds scales with their respective laminar flame speeds [28]. The location of the centroid is the heat-release weighted center of chemiluminosity. From the radial and axial coordinates, the angles $\alpha$ can be determined. For the inner enrichment case (Flame 3, Table 1), we assume that the lean mixture burns at the flame speed of the lean flammability limit of 0.45 [29]. For the D-shaped flame, with a corner recirculation zone, we assume that only the inner part of the flame contributes to the fluctuations, as a V-flame. The angle and centroid are determined from the images.

The geometric characterization allows us to obtain an area perturbation for each case. The overall perturbation to the heat release rate is then worked out as the sum of the perturbations to each branch, weighted by the corresponding fuel fraction burned in that particular region. In other words, the total heat release rate perturbation is a weighted sum of relative perturbations of the separate branches of premixed flame bases divided by its mean 
power.

$$
\begin{array}{r}
F^{T}=\left(\sum_{i=1}^{N} \hat{Q}_{i} / \bar{Q}\right) /(\hat{u} / \bar{u})=\sum_{i=1}^{N} F_{i} Q_{i} / \bar{Q} \\
=\left(\sum_{i=1}^{N} F_{i} X_{i} \bar{Q}\right) / \bar{Q}=\sum_{i=1}^{N} F_{i} X_{i}
\end{array}
$$

where $X_{i}=Q_{i} / \bar{Q}$, is the ratio of the heat release of each flame branch to the total heat release.

\section{Experimental data and comparison with theoretical results}

Figure 3 shows the mean unforced flame structure for all tested cases, as measured by chemiluminescence. The top half of each panel shows the lineof-sight measurements, while the bottom panels show the radius weighted Abel-deconvoluted images. Using the assumption that the overall rate of heat release is proportional to local chemiluminescence integrated over the volume, the centroid of heat release rate becomes the centroid of the radiusweighted Abel deconvoluted images. A threshold of $70 \%$ of maximum intensity was used to determine the centroid, to avoid weighting of the surrounding noise. The distribution of chemiluminescence is clearly significantly affected by both stratification ratio as well as global equivalence ratio. The length of the flame decreases from outer to inner stratification, while its distribution becomes more concentrated (left to right). The overall equivalence ratio (top to bottom) also leads to more compact flames for premixed and outer stratification $(S R=0.5)$, whilst having little effect on the inner stratified flame. The choice of model was based on the assessment of both averaged as well as individual images in the high speed measurements. A detailed examination of the images was made at higher magnification, to identify how the flames are stabilized and attached. The resulting model geometries are indicated on the panels in Fig. 3 and last column in Table 1. The A-flame description is suitable for the richer global equivalence ratios $(\Phi=0.7$ and 0.8$)$ with leaner inner streams $(S R=0.5)$, as well as the premixed case at $\Phi=0.7$. One unified flame branch appears in most cases, attaching to both inner and outer tubes as the flame gets richer. Each branch is assumed to burn at the corresponding inner or outer equivalence ratio. The M-flame fits the images for all inner stratified flames $(S R=2.0)$ and the premixed flame with the richest global 
equivalence ratio $(\Phi=0.8)$. These flames show two clear branches of flame in $\mathrm{OH}^{*}$ chemiluminescence images, and two additional anchoring points at the edge of inner and outer tube. The D-flame is assigned to the premixed flame $(S R=1.0)$ at lean global equivalence ratio $\left(\Phi_{g}=0.6\right)$ : the flame stabilizes along the edge of bluff body with a developed corner recirculation zone. The corresponding flame models are shown in Fig. 3 as well as the last column in Table 1.

The experimental gain and phase differences in the linear amplitude range are shown in Fig. 4. The values shown are for acoustic input velocity measurements determined from the two-microphone method (TMM), along with the values obtained from the model. The top right subfigure Fig. $4\left(\Phi_{g}=0.6\right.$, $S R=2.0$ ) shows typical values and variances for FTFs based on the TMM as well as HWA, obtained by determining the variance over time blocks of 0.5 $\mathrm{s}$ from the original $4 \mathrm{~s}$ record. Velocity values obtained with HWA are higher in general than with TMM, as the former detect both turbulent fluctuations and vortical disturbances from the swirlers. Thus, the corresponding FTF values for the HWA are lower than the those for the TMM, which are here used as the standard. An analysis of the frequency spectrum of the PMT signals (not shown here) indicates that the normalized fluctuation in heat release rate has rather high amplitude at low forcing frequencies, even when unforced. This background noise leads to the very high gains in FTF at frequencies below $100 \mathrm{~Hz}$. The values for the gain of the premixed $(S R=1.0)$ and outer enriched stratified flames $(S R=0.5)$ are similar, as are their flame shapes: the gain reaches a peak above two at low frequency, and decreases gradually as the forcing frequency increases at a lean global equivalence ratio of 0.6. As $\Phi_{g}$ increases to 0.7 and 0.8 , distinctive peaks and nodes appear in the gain. The inner enriched flame $(S R=2.0)$ is significantly more sensitive to velocity fluctuations. For the three cases of global equivalence ratio, the gain for the inner enriched flame increases to a value above 3, reaching a peak, then decreasing towards a node around $160 \mathrm{~Hz}$, and a second peak around $220 \mathrm{~Hz}$, before finally decreasing to below unity at higher frequencies. The appearance of nodes with frequency is typical of situations in which two phenomena with different time scales interfere. In the present case, this is associated with the interference of the direct axial acoustic wave and the convection of the swirl-induced perturbation. The phase difference between heat release rate and velocity are predictably higher in the case of the longer premixed and outer stratified flames than for the shorter flames with inner enrichment or higher global equivalence ratios. 
Model results are shown as dashed lines on the same figure. A global fit to the model parameters yields $\zeta=\chi=-1$ for all cases, and the offset phase delay in the swirler is taken as $\theta_{0 s}=-1$ radian. For each flame, a mean centroid length $L_{f}$ and angle $\alpha$ are obtained to yield the final model gain and phase curves, which are in reasonable agreement with the experimental results. However, there are some glaring discrepancies: gains for the premixed flames at higher equivalence ratios are clearly not captured at low frequencies, and the models predict a more jagged behaviour for the gain than the simple geometrical model implies. The peaks and troughs in the models are a result of the time difference between convection to the flame and passage through the swirlers. The tight spacing in the oscillations compared to the experiments suggests that the time difference associated with vortical disturbances is not perfectly captured, and the variations with frequency are significantly attenuated. The phase is less dependent on the details, however, possibly because it is primarily dependent on the measured time delays, which are a direct function of the measured flame length $L_{f}$.

The flame heat release response of premixed and stratified flames is a complex function of the location and phasing of the heat release rate, so it is rather comforting that a very simple model based on geometric assumptions is actually able to give credible results.

\section{Conclusion}

We have measured the low amplitude linear response of lean-premixed swirl-stabilized flames to acoustic forcing over a range of equivalence ratios and stratifications. A model based on the $G$-equation and geometric characteristics of the flame front is used to described the response of the flame in comparison with the experimental measurement. The effect of the swirlers appears as an additive effect via a transfer function of the velocity disturbances, and the effect of stratification is accounted for by changes in the flame speed. The comparison of model and experiments shows that it is possible to extend the use of the adapted $G$-equation model to the present flames with good agreement, but that the more complex shape and behavior of turbulent stratified flames may require yet more realistic flame geometry models to account for their full behavior. 


\section{Acknowledgments}

The authors acknowledge the support provided by the Cambridge Over-

seas Trust and China Scholarship Council. Additional funding was provided by Rolls-Royce plc for the initial set up of the experiments.

\section{References}

[1] T. C. Lieuwen, V. Yang, Combustion instabilities in gas turbine engines: operational experience, fundamental mechanisms and modeling, American Institute of Aeronautics and Astronautics, 2005.

[2] Y. Huang, V. Yang, Progress in Energy and Combustion Science 35 (2009) 293-364.

[3] S. Candel, Proceedings of the combustion institute 29 (2002) 1-28.

[4] Y. Huang, S. Wang, V. Yang, AIAA Journal 44 (2006) 724-740.

[5] T. Schuller, D. Durox, S. Candel, Combustion and Flame 135 (2003) $525-537$.

[6] B. D. Bellows, Y. Neumeier, T. Lieuwen, Journal of Propulsion and Power 22 (2006) 1075-1084.

[7] J. H. Cho, T. Lieuwen, Combustion and Flame 140 (2005) 116-129.

[8] A. Birbaud, S. Ducruix, D. Durox, S. Candel, Combustion and Flame 154 (2008) 356-367.

[9] T. Lieuwen, Y. Neumeier, B. Zinn, Combustion Science and Technology 135 (1998) 193-211.

[10] R. Balachandran, B. Ayoola, C. Kaminski, A. Dowling, E. Mastorakos, Combustion and Flame 143 (2005) 37-55.

[11] T. Sattelmayer, Journal of Engineering for Gas Turbines and Power 125 (2003) 11-19.

[12] T. Scarinci, C. Freeman, I. Day, in: Proceedings of ASME Turbo Expo 2004: Power for Land, Sea, and Air, June 14-17, 2004, Vienna, Austria, ASME Paper No. GT2004-53767. 
[13] K. Kim, S. Hochgreb, Combustion and Flame 158 (2011) 2482-2499.

[14] K. T. Kim, S. Hochgreb, Combustion Science and Technology 184 (2012) 608-628.

[15] T. Lieuwen, Journal of Propulsion and Power 19 (2003) 765-781.

[16] A. Dowling, Journal of Fluid Mechanics 346 (1997) 271-290.

[17] A. Dowling, Journal of Fluid Mechanics 394 (1999) 51-72.

[18] S. Ducruix, T. Schuller, D. Durox, S. M. Candel, Journal of Propulsion and Power 19 (2003) 722-734.

[19] N. Noiray, D. Durox, T. Schuller, S. Candel, Journal of Fluid Mechanics 615 (2008) 139-167.

[20] T. Schuller, D. Durox, S. Candel, Combustion and Flame 134 (2003) $21-34$.

[21] P. Palies, T. Schuller, D. Durox, S. Candel, Proceedings of the Combustion Institute 33 (2011) 2967-2974.

[22] B. Higgins, M. McQuay, F. Lacas, S. Candel, Fuel 80 (2001) 1583-1591.

[23] C. J. Dasch, Applied Optics 31 (1992) 1146-1152.

[24] N. Peters, Journal of Fluid Mechanics 242 (1992) 611-629.

[25] P. Palies, D. Durox, T. Schuller, S. Candel, Combustion and Flame 157 (2010) 1698-1717.

[26] D. Shin, T. Lieuwen, Journal of Fluid Mechanics 721 (2013) 484-513.

[27] N. A. Cumpsty, F. E. Marble, Proceedings of the Royal Society A: Mathematical, Physical and Engineering Sciences 357 (1977) 323-344.

[28] Y. Dong, C. M. Vagelopoulos, G. R. Spedding, F. N. Egolfopoulos, Proceedings of the Combustion Institute 29 (2002) 1419-1426.

[29] A. Levy, Proceedings of the Royal Society A: Mathematical, Physical and Engineering Sciences 283 (1965) 134-145. 


\section{List of Tables}

1 Test conditions for premixed and stratified flames. Last column discussed in Section 3. . . . . . . . . . . . . . . . . 15 


\begin{tabular}{|c|c|c|c|c|c|}
\hline Flame & $\Phi_{g}$ & $S R$ & $\Phi_{i}$ & $\Phi_{o}$ & Model \\
\hline 1 & \multirow{3}{*}{0.6} & 1.0 & 0.60 & 0.60 & $\mathrm{D}$ \\
\hline 2 & & 0.5 & 0.36 & 0.72 & $\mathrm{D}$ \\
\hline 3 & & 2.0 & 0.89 & 0.45 & $\mathrm{M}$ \\
\hline 4 & \multirow{3}{*}{0.7} & 1.0 & 0.70 & 0.00 & $\mathrm{~A}$ \\
\hline 5 & & 0.5 & 0.42 & 0.84 & $\mathrm{~A}$ \\
\hline 6 & & 2.0 & 1.04 & 0.52 & $\mathrm{M}$ \\
\hline 7 & \multirow{3}{*}{0.8} & 1.0 & 0.80 & 0.80 & $\mathrm{M}$ \\
\hline 8 & & 0.5 & 0.48 & 0.91 & A \\
\hline 9 & & 2.0 & 1.19 & 0.60 & M \\
\hline
\end{tabular}

Table 1: Test conditions for premixed and stratified flames. Last column discussed in Section 3 . 


\section{List of Figures}

1 Schematic of the stratified swirl burner. Dimensions in millimeters, not to scale. Area ratio of inner channel to outer channel, $A_{i} / A_{o}=0.512$. . . . . . . . . . . . . . . . . 17

2 Schematic of flame geometry based on inlet conditions. . . . . 18

3 Average self-normalized $\mathrm{OH}^{*}$ chemiluminescence images split into two panels for each case, line-of-sight (top) and radialweighted Abel transformed (bottom). The letters refer to the geometric model used for each flame . . . . . . . . . . . . 19

4 Comparison of experimental and theoretical results of FTF. Variance of TMM and HWA data are shown in the top right subfigure with inlet condition of $\Phi_{g}=0.6, \mathrm{SR}=2.0$. Phase

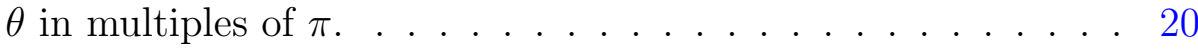




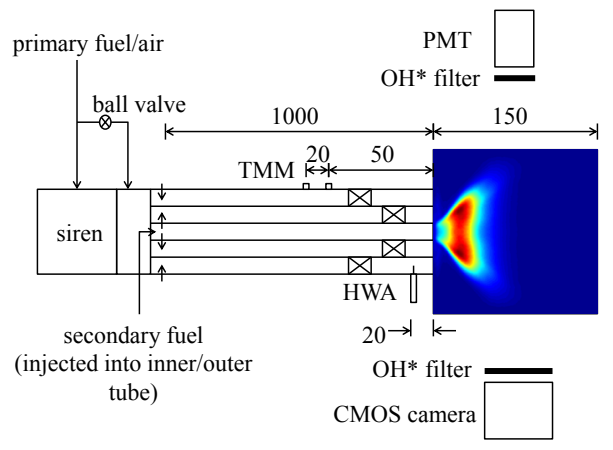

Figure 1: Schematic of the stratified swirl burner. Dimensions in millimeters, not to scale. Area ratio of inner channel to outer channel, $A_{i} / A_{o}=0.512$. 


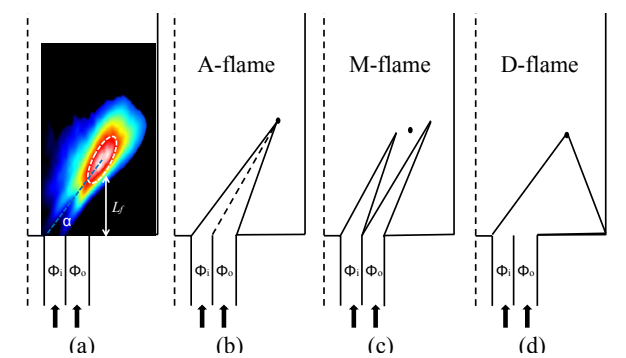

Figure 2: Schematic of flame geometry based on inlet conditions. 


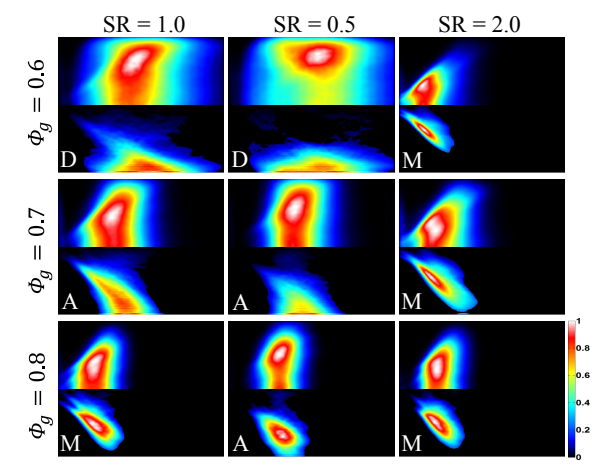

Figure 3: Average self-normalized $\mathrm{OH}^{*}$ chemiluminescence images split into two panels for each case, line-of-sight (top) and radial-weighted Abel transformed (bottom). The letters refer to the geometric model used for each flame 


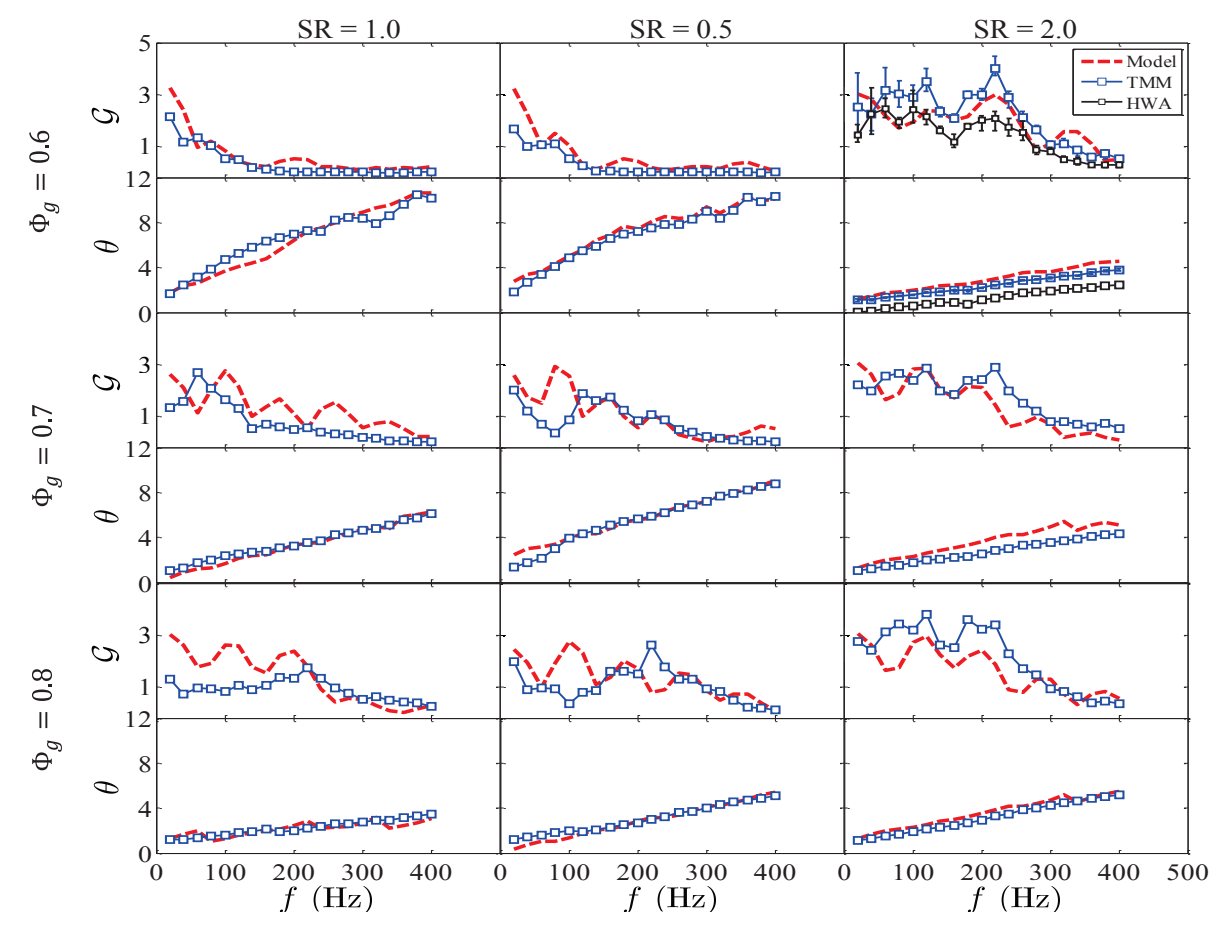

Figure 4: Comparison of experimental and theoretical results of FTF. Variance of TMM and HWA data are shown in the top right subfigure with inlet condition of $\Phi_{g}=0.6, \mathrm{SR}$ $=2.0$. Phase $\theta$ in multiples of $\pi$. 